\title{
Association between DRD4 gene polymorphism and personality variation in great tits: a test across four wild populations
}

\author{
PETER KORSTEN,$*{ }^{* 1}$ JAKOB C. MUELLER, ${ }^{*}$ CHRISTINE HERMANNSTÄDTER, * KAREN M. \\ BOUWMAN,$\dagger^{\prime 2}$ NIELS J. DINGEMANSE,$\dagger^{3}{ }^{3}$ PIET J. DRENT, $\ddagger$ MIRIAM LIEDVOGEL,,${ }^{\prime 4}$ ERIK \\ MATTHYSEN,§ KEES VAN OERS, $\ddagger$ THIJS VAN OVERVELD,§SAMANTHA C. PATRICK, $\uparrow$ JOHN L. \\ QUINN, BEN C. SHELDON, JOOST M. TINBERGEN+ and BART KEMPENAERS* \\ *Department of Behavioural Ecology E Evolutionary Genetics, Max Planck Institute for Ornithology, Seewiesen, Germany, \\ tAnimal Ecology Group, Centre for Ecological and Evolutionary Studies, University of Groningen, The Netherlands, \\ $\ddagger$ Department of Animal Population Biology, Netherlands Institute of Ecology (NIOO-KNAW), The Netherlands, §Evolutionary \\ Ecology Group, Department of Biology, University of Antwerp, Wilrijk, Belgium, Edward Grey Institute, Department of \\ Zoology, University of Oxford, Oxford, UK
}

\begin{abstract}
Polymorphisms in the dopamine receptor D4 gene (DRD4) have been related to individual variation in novelty-seeking or exploratory behaviour in a variety of animals, including humans. Recently, the human DRD4 orthologue was sequenced in a wild bird, the great tit (Parus major) and a single nucleotide polymorphism in exon 3 of this gene (SNP830) was shown to be associated with variation in exploratory behaviour of labraised individuals originating from a single wild population. Here we test the generality of this finding in a large sample of free-living individuals from four European great tit populations, including the originally sampled population. We demonstrate that the association between SNP830 genotype and exploratory behaviour also exists in freeliving birds from the original population. However, in the other three populations we found only limited evidence for an association: in two populations the association appeared absent; while in one there was a nonsignificant tendency. We could not confirm a previously demonstrated interaction with another DRD4 polymorphism, a 15 bp indel in the promoter region (ID15). As yet unknown differences in genetic or environmental background could explain why the same genetic polymorphism (SNP830) has a substantial effect on exploratory behaviour in one population, explaining $4.5-5.8 \%$ of the total variance-a large effect for a single gene influencing a complex behavioural trait-but not in three others. The confirmation of an association between SNP830 genotype and personality-related behaviour in a wild bird population warrants further research into potential fitness effects of the polymorphism, while also the population differences in the strength of the association deserve further investigation. Another important future challenge is the identification of additional loci influencing avian personality traits in the wild.

Keywords: animal personality, candidate gene, dopamine receptor, $D R D 4$, genetic association, exploratory behaviour, gene by environment interaction, great tit Parus major, novelty seeking, replication study, wild populations
\end{abstract}

Received 19 August 2009; revision 16 October 2009; accepted 28 October 2009

\footnotetext{
Correspondence: Bart Kempenaers, Fax: +49 (0) 8157932 400; E-mail: b.kempenaers@orn.mpg.de

${ }^{1}$ Present address: Institute of Evolutionary Biology, University of Edinburgh, UK.

${ }^{2}$ Present address: Department of Biological Sciences, Virginia Tech, VA, USA.

${ }^{3}$ Present address: Department of Behavioural Ecology \& Evolutionary Genetics, Max Planck Institute for Ornithology, Seewiesen, Germany.

${ }^{4}$ Present address: Animal Ecology, Department of Ecology, Lund University, Lund, Sweden.
} 


\section{Introduction}

In animals, consistent individual differences in suites of correlated behaviour occur, similar to personality variation in humans (Gosling 2001; Sih et al. 2004a,b; Réale et al. 2007). Historically, behavioural ecologists have often dismissed such behavioural variation as random noise around an adaptive mean (Wilson 1998). However, there is now growing interest in the idea that variation in behavioural phenotypes is adaptive, with different personalities having a selective advantage under different circumstances (Dall et al. 2004; Wolf et al. 2007, 2008; Biro \& Stamps 2008; McNamara et al. 2009). Such personality-related behavioural variation could have important ecological and evolutionary consequences (Sih et al. 2004a,b; Dingemanse \& Réale 2005; Réale et al. 2007) and may, for example, predict success in exploiting novel food sources, colonization of new habitats, or adaptation to environmental change (e.g. Greenberg 1990; Sol et al. 2002; Martin \& Fitzgerald 2005; Shultz et al. 2005; Duckworth \& Badyaev 2007; Harfmann Short \& Petren 2008). Yet, the molecular genetic basis that may underlie part of this variation is almost completely unknown.

Both in humans and other animal species, personality variation has a substantial heritable component (McGue \& Bouchard 1998; van Oers et al. 2005; Réale et al. 2007). It is largely unclear, however, what selective processes contribute to the maintenance of this genetic variation. The observed genetic variation may simply be the result of mutation-selection balance, but it is more likely that adaptive selective processes, such as frequency- and density-dependent selection for different personality types and/or balancing selection due to spatio-temporal environmental variation, contribute to its maintenance (Wilson 1998; Dall et al. 2004; Dingemanse et al. 2004; Réale et al. 2007; Quinn et al. 2009; see also Mitchell-Olds et al. 2007). Insight into the molecular genetic mechanisms underlying personality variation, specifically in wild populations, would help to improve our understanding of the evolutionary processes that promote and maintain such variation (van Oers et al. 2005; see also Ellegren \& Sheldon 2008). For example, the identification of genetic loci underlying personality variation in wild populations opens the unique possibility of investigating how frequencies of specific variants of personality-related genes change in space and time in relation to environmental variation (i.e. to observe selection on these genes; Ellegren \& Sheldon 2008). This would thus make it possible to directly link selection on the phenotypic level to changes in the observed genetic variation.

One of the most promising candidate genes related to personality variation in humans is the dopamine receptor D4 gene (DRD4; Savitz \& Ramesar 2004). $D R D 4$ is expressed in the central nervous system as part of the dopaminergic system involved in motivational behaviours (Netter 2006), and has been associated with variation in novelty seeking (Munafò et al. 2003, 2008; Savitz \& Ramesar 2004). Recently, Fidler et al. (2007) identified the orthologue of the human DRD4 in a wild bird species-the great tit (Parus major) - that is a commonly used model in ecological field studies. Fidler and colleagues furthermore detected a single nucleotide polymorphism located in exon 3 of this orthologue (SNP830, with a C-T substitution) that associated with individual variation in exploratory behaviour, both in lines artificially selected for divergent exploratory behaviour and in wildcaught nestlings which were raised in the lab. In addition, Fidler et al. (2007) found that in the wild-caught birds there was a significant interaction of the SNP830 genotype with another DRD4 polymorphism-a $15 \mathrm{bp}$ indel (ID15) located $5^{\prime}$ to the putative transcription initiation site. Associations between polymorphisms of DRD4 orthologues and novelty seeking-related behaviours have also been found in domestic chickens Gallus gallus (Flisikowski et al. 2009), domestic horses Equus caballus (Momozawa et al. 2005), domestic dogs Canis familiaris (Hejjas et al. 2007), and captive vervet monkeys Chlorocebus aethiops (Bailey et al. 2007; James et al. 2007). However, unlike the great tit, none of these species is suitable for large-scale field studies on wild populations.

The great tit has proven an excellent model in ecological and evolutionary research in general (e.g. Postma \& van Noordwijk 2005; Charmantier et al. 2008) and is probably the best studied model for the ecology and evolution of personality variation in the wild (Sih et al. 2004a; Réale et al. 2007; see Groothuis \& Carere 2005 for a review). Individual great tits differ consistently in their exploratory behaviour along an axis of fast versus slow exploration when measured in a standard test room representing a novel environment (Verbeek et al. 1994; Dingemanse et al. 2002). The variation in exploratory behaviour is phenotypically and genetically correlated with several other behaviours: faster explorers are relatively bolder towards novel objects, more aggressive towards conspecifics, take more risks, and form foraging routines more quickly (Verbeek et al. 1994, 1996; van Oers et al. 2004a,b). Both artificial selection experiments and field studies (e.g. using parent offspring regression as well as 'animal model' methodology) have shown a heritable basis for the variation in exploratory behaviour in multiple populations $\left(h^{2}=0.22-0.54\right.$; Dingemanse et al. 2002; Drent et al. 2003; Quinn et al. 2009). Fidler et al. (2007) subsequently found that faster exploration was associated with the presence of the $\mathrm{T}$ 
allele for SNP830. Finally, field studies presented evidence for fitness effects of individual differences in exploratory behaviour through-often spatially and/or temporally varying or otherwise context-specific-associations with breeding success (Dingemanse et al. 2004; Both et al. 2005; Quinn et al. 2009), extra-pair mate choice (van Oers et al. 2008), and survival (Dingemanse et al. 2004).

In the present paper our overall aim is to test the generality of the association between the DRD4 SNP830 C/T polymorphism and exploratory behaviour across different wild great tit populations. We specifically aimed to: (i) test if the association between the DRD4 SNP830 genotype and exploratory behaviour found in captive, hand-raised great tits by Fidler et al. (2007) is also present in free-living individuals from the same wild population; (ii) test if the association between $D R D 4$ genotype and exploratory behaviour is present in three other wild great tit populations; (iii) confirm the previously reported effect on exploratory behaviour of the interaction of the SNP830 with the ID15 indel.

\section{Materials and methods}

\section{Study populations and sample selection}

The populations investigated for exploratory behaviour and DRD4 SNP830 and ID15 genotypes are: Westerheide (WH; The Netherlands; $n=79$ ), Lauwersmeer (LM; The Netherlands; $n=196)$, Boshoek (BH; Belgium; $n=103$ ), and Wytham Woods (WW; United Kingdom; $n=288$ ) (Fig. 1; Table S1, Supporting Information). For more details on these study populations see Dinge- manse et al. (2002; WH), Nicolaus et al. (2009; LM), Hollander et al. (2008; BH), and McCleery et al. (2004; WW). The unselected captive individuals in which Fidler et al. (2007) found an association between polymorphisms of the DRD4 gene and exploratory behaviour originated from the Westerheide population where they were collected as nestlings (in the spring of 1998) and subsequently hand-raised and tested for exploratory behaviour when juveniles (for details on the test protocol see also Drent et al. 2003).

We only included first-year birds that had not yet bred and were caught during the nonbreeding season between July-March to reduce potential variation in behaviour among individuals due to differences in age and (breeding) experience. These criteria also kept our sample relatively comparable to the sample of handraised juvenile birds investigated by Fidler et al. (2007). Birds born in the study areas were all individually ringed as nestlings and their exact age was known; birds not born in the study areas $(50.3 \%$ of the total sample of 666) were aged as first-year based on the colour of their primary wing coverts (Jenni \& Winkler 1994). For the Lauwersmeer, Boshoek and Wytham Woods populations, only one offspring from each brood was included (where brood of origin was known), as well as all immigrants that entered the study population. In Lauwersmeer and Wytham Woods, data were collected in two and three nonbreeding seasons respectively (see Table S1, Supporting Information), and we only included one randomly chosen individual for each set of birds with a parent-offspring relationship. In summary, no individual had a known first-order family relationship to any other individual in the Lauwersmeer, Boshoek and Wytham Woods datasets. For

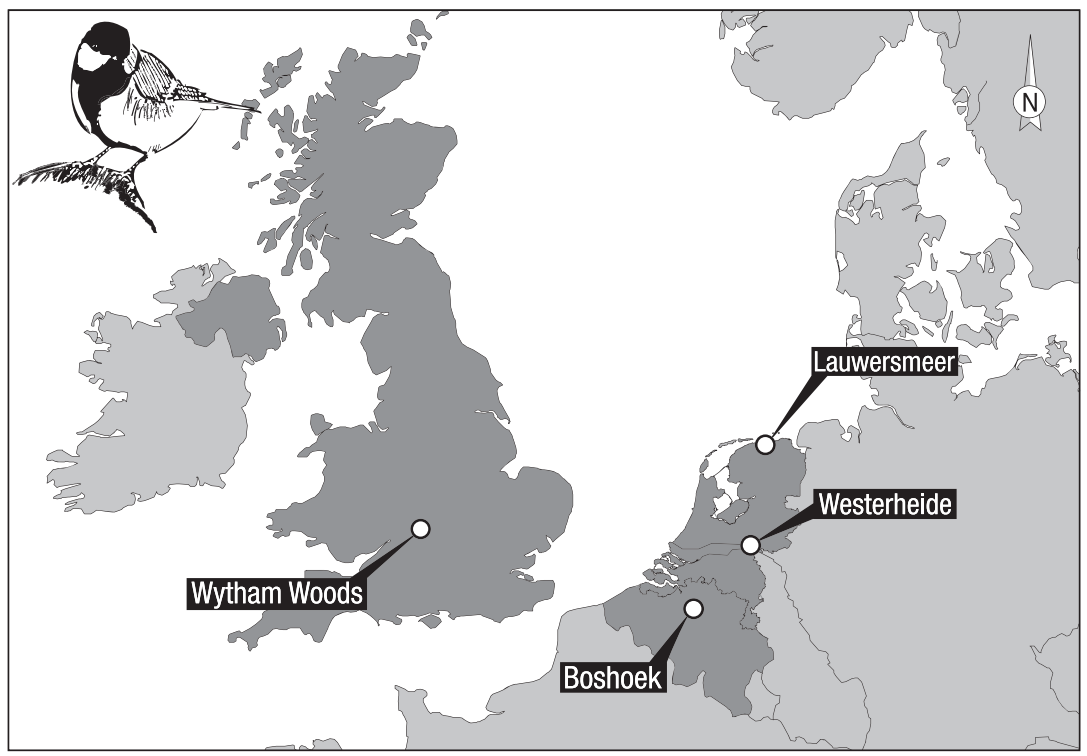

Fig. 1 Locations of four wild great tit populations investigated for associations between exploratory behaviour and DRD4 SNP830 and ID15 polymorphisms. Populations are Westerheide (WH), Lauwersmeer (LM), Boshoek (BH) and Wytham Woods (WW). 
Westerheide multiple individuals from the same brood were included to obtain sufficient sample size. We took the relatedness of birds in the Westerheide data set into account in our statistical analyses (see below).

\section{Measuring exploratory behaviour}

The exploratory behaviour of individuals was measured following a well-established protocol using a standard test chamber with five artificial 'trees' as a novel environment (see Dingemanse et al. 2002). In summary, individuals were caught with mistnets near feeding stations or when roosting in nestboxes, and transported to the lab where they were kept overnight in individual cages adjacent to the test chamber. The next morning each individual was released from its cage directly into the test chamber by opening a sliding door. After release, the individual's behaviour was monitored for $2 \mathrm{~min}$ and all hops and flights between perches (e.g. branches of the artificial trees, sliding doors, the walls and floor) were recorded. Exploration scores were calculated by summing all recorded hops and flights per individual. Procedures were standardized between populations and the test chambers had similar design and dimensions in all four populations $(\mathrm{WH}, \mathrm{LM}, \mathrm{BH}$ : $4.0 \times 2.4 \times 2.3 \mathrm{~m}$; WW: $4.0 \times 3.3 \times 2.5 \mathrm{~m}$ ). In the Wytham Woods population, the observation protocol differed slightly in that hops within a single perch (e.g. branch of a tree or floor) were also included. These additional hops could not be excluded from the exploration score retrospectively due to the methodology of data collection (see also Quinn et al. 2009). It is unlikely that this difference between test protocols qualitatively affected our results and conclusions, because previous work has shown that small modifications of this type of behavioural test protocols give qualitatively similar results (Dingemanse et al. 2002; Quinn et al. 2009). The majority of tests were carried out between 8:00-13:00 h in all four populations. All tests included in our analyses are of birds that were tested for the first time during their lives, thus excluding any effects of habituation to the test as have been reported previously (Dingemanse et al. 2002).

Fidler et al. (2007) used a somewhat different protocol to assess exploratory behaviour. In short, birds were raised in the lab under standard conditions and tested between ca. 35-50 days after hatching (Drent et al. 2003). Fidler and colleagues furthermore used a compound measure for exploratory behaviour ('early exploratory behaviour' or 'EEB') that included the exploration of a novel test room (using a modified observation protocol compared to ours) and the approach to two different novel objects (for details see
Verbeek et al. 1994; Drent et al. 2003). Our protocol for assessing exploratory behaviour was modified compared to the protocol used in Fidler et al.'s study to make it suitable for reliably testing wild-caught adults (following Dingemanse et al. 2002).

\section{SNP830 and ID15 genotyping}

We collected blood (WH, LM and WW) or feather samples $(\mathrm{BH})$ for DNA extraction after the exploration test of each individual. Blood samples were stored in either Queen's lysis buffer $(\mathrm{WH})$, or pure ethanol (LM, WW); feathers were stored in paper envelopes (BH). DNA was extracted from $\mathrm{WH}$ and LM blood samples using commercially available kits: the GFX Genomic Blood DNA Purification Kit (GE Healthcare, Freiburg, Germany) for samples in Queen's buffer (WH), and the DNeasy Blood \& Tissue Kit (Qiagen, Hilden, Germany) for samples in ethanol (LM). DNA from WW samples was extracted using a standard Chelex extraction protocol (Walsh et al. 1991). Phenol-chloroform extraction was used to obtain DNA from the feathers $(\mathrm{BH})$.

The genotyping for the SNP830 (DRD4 C/T SNP830 of Genbank entry DQ006802) of BH, LM and WH samples was performed through a PCR based primer extension reaction and detection of the allele-specific extension products by matrix-associated laser desorption/ionization time of flight (MALDI-TOF) mass spectrometry (Sequenom) at the Clinic for Psychiatry and Psychotherapy of the Technical University, Munich (Germany). WW samples were SNP830 genotyped at the Edward Grey Institute of the University of Oxford (UK) following the six-FAM labelled primer combination design and amplification protocol previously published (Fidler et al. 2007). A sample of $165 \mathrm{WW}$ birds was independently genotyped for SNP830 in the lab in Munich, which showed a discrepancy in the assigned genotypes for only two individuals $(1.2 \%$ of $n=165$; these two individuals were both assigned $\mathrm{C} / \mathrm{T}$ in Oxford, while they were assigned $\mathrm{T} / \mathrm{T}$ in Munich); hence we believe the genotyping error rate to be low. A total of 655 birds were successfully genotyped for SNP830. Samples were genotyped for the ID15 insertion-deletion polymorphism (DRD4 ID15 indel, $\pm 15 \mathrm{bp}$, coordinates 713-727 of Genbank entry DG006801) through amplification of the region using primers DR902R and DR634F with sequences GCC CCA AAG TTC CCT TAC TCT T and CCT CTG GAA GCA GAA TTT GAG GA, respectively (Fidler et al. 2007). Amplification products were subsequently resolved using an ABI PRISM 3100 Genetic Analyzer (Applied Biosystems) along with a molecular size standard (GeneScan-500 LIZ, Applied Biosystems) and amplification product sizes were calculated using the 
commercial software GENESCAN 3.7 and GENOTYPER 3.6 (Applied Biosystems). The DRD4 ID15+ and ID15alleles generated amplification products of 269 and $254 \mathrm{bp}$, respectively. A total of 491 birds were successfully genotyped for ID15. All birds were sexed using Griffiths et al.'s (1998) P2 and P8 primers.

\section{Statistical analysis}

We used Chi-square tests to test for Hardy-Weinberg equilibrium in each of the populations and to test whether genotype frequencies differed between the populations. To investigate the association between the DRD4 SNP830 genotype and exploratory behaviour we tested for an additive genetic effect of the SNP830 alleles ('additive genetic model') and for a dominance effect of the $\mathrm{T}$ allele ('dominant-T genetic model') following Fidler et al. (2007). Similarly for ID15, we tested for an additive genetic effect and a dominant ID15effect (see Fidler et al. 2007). In addition, we tested if there was a significant effect of the SNP830 $\times$ ID15 interaction, as was previously found in the Westerheide population (Fidler et al. 2007). We used a general linear mixed model approach and took into account the family clusters in the Westerheide population by including brood of origin (brood ID) as a random factor. For direct comparison, we included the original data of the birds studied by Fidler et al. (2007) that were collected as nestlings in the Westerheide $(n=91)$ in some of our analyses; we also took into account the family clusters in this data set using the above approach. Significance (two-tailed $\alpha=0.05$ ) was assessed by the increase in deviance ( $\Delta$ deviance, which follows a $\chi^{2}$ distribution) when a parameter was removed from the model. The association analyses were carried out with the MLwiN 2.02 software package, which is particularly suited for implementing multilevel and mixed models (Rasbash et al. 2004; estimation method was set to IGLS).

Exploration scores are known to vary within individuals with season (Dingemanse et al. 2002), and the slopes of these within-individual changes did not differ between the four populations tested (Bouwman et al. submitted). Therefore, we corrected the exploration scores for the seasonal trend for all four populations using the equation: 'season corrected exploration score' $=$ 'measured exploration score' - $(0.030 \times$ 'July date' $^{\prime}+10$, where 'July date' was the number of days from 1 July onwards. For details see Dingemanse et al. (2002) and Bouwman et al. (submitted).

After separate analyses for each population, we combined the data of the four populations to maximize the statistical power for detecting an overall association between DRD4 genotype and exploratory behaviour, and to test for population differences in the strength of the association. As there were differences in the magnitude of exploration scores between the study populations (see Results), we standardized the scores for each population by subtracting the population mean and dividing by the population standard deviation before inclusion in the models. We also standardized Fidler et al.'s exploration scores following the same procedure before including these data in our models.

\section{Results}

None of the populations deviated from Hardy-Weinberg equilibrium for either the SNP830 (Fig. 2A) or ID15 (Fig. 2B) polymorphism (all $P>0.47$; see Table S2, Supporting Information). The observed genotype frequencies did not differ between the populations, either for SNP830 ( $\chi^{2}=4.986$, d.f. $\left.=6, n=655, P=0.55\right)$ or for ID15 $\left(\chi^{2}=6.926\right.$, d.f. $\left.=6, n=491, P=0.33\right)$. In contrast, mean exploration scores differed significantly between populations (Fig. 3; $\Delta$ deviance $=64.434$, d.f. $=3, n=666, P<0.001)$.

In the Westerheide population, exploratory behaviour was significantly associated with SNP830 genotype for the additive genetic model, while the dominant-T model showed an almost significant trend (Fig. 4A; Table 1), which is largely consistent with the findings of Fidler et al. (2007). In this population SNP830 genotype explained $5.8 \%$ and $4.5 \%$ of the total variance in exploration scores for the additive and dominant genetic models, respectively. In Lauwersmeer (Fig. 4B), a similar but weaker and marginally non-significant association occurred for the dominant-T model only (Table 1B). Exploratory behaviour was not significantly associated with SNP830 genotype in Boshoek or Wytham Woods (Fig. 4C, D; Table 1). The association between the SNP830 genotype and exploratory behaviour was independent of the sex of individuals, as sex and its interaction with SNP830 had no significant effect on exploratory behaviour in any of the populations (all $P>0.17$; see Table S3, Supporting Information). Therefore, we pooled the sexes in further analyses.

Subsequent analyses showed that the association between SNP830 genotype and exploratory behaviour was similar for Fidler et al.'s (2007) and our data of the Westerheide. SNP830 genotype explained 5.6\% and $6.0 \%$ of the total variance in exploratory behaviour in Fidler et al.'s dataset for the additive and dominant genetic models, respectively. Furthermore, there was no significant interaction between SNP830 genotype and the origin of the data (SNP830 genotype $\times$ Fidler et al.'s vs. our data; additive genetic model: $n=168, \Delta$ deviance $=1.348$, d.f. $=1, \quad P=0.25$, dominant-T genetic model: $n=168, \Delta$ deviance $=0.037$, d.f. $=1, P=0.85$ ). 

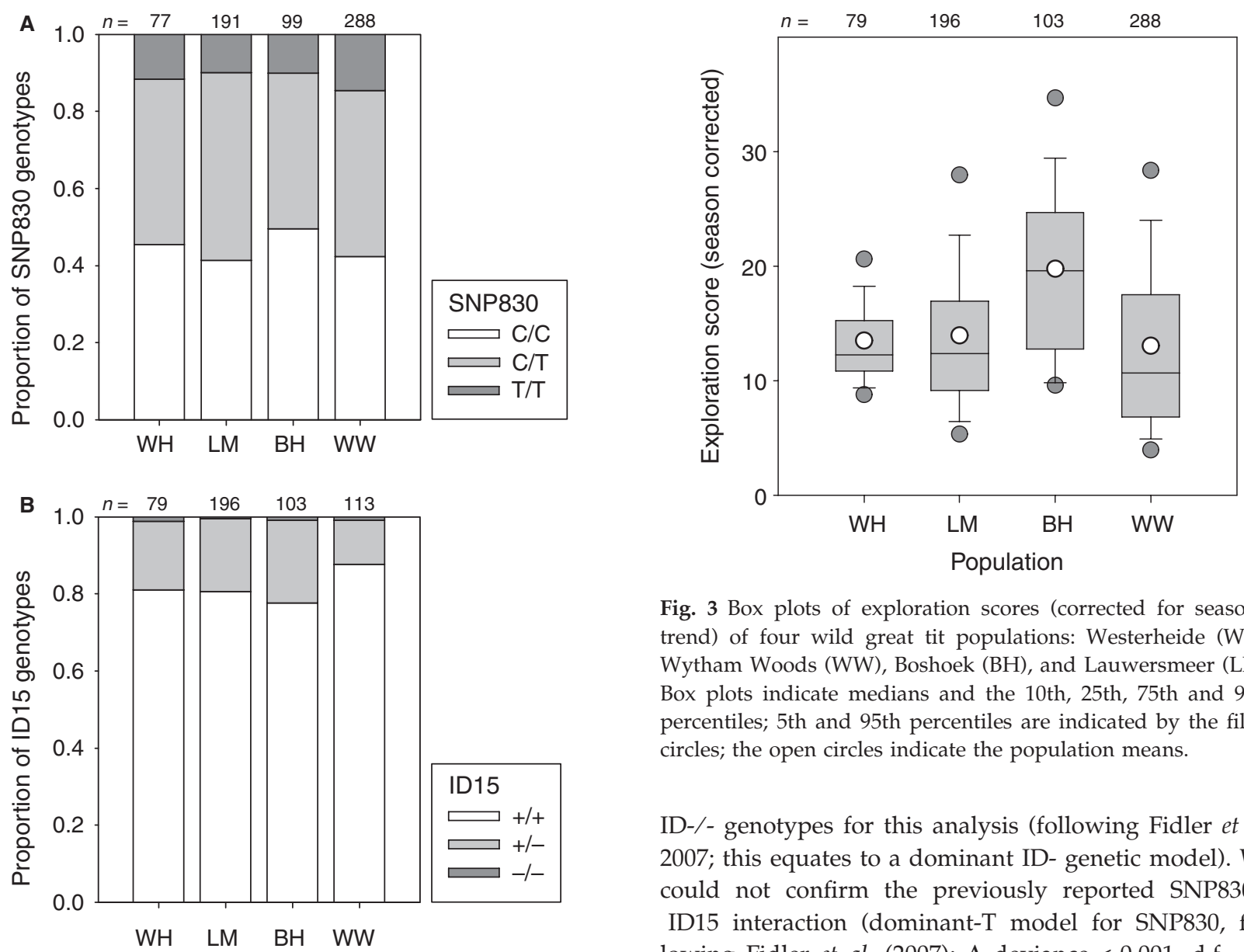

Fig. 3 Box plots of exploration scores (corrected for seasonal trend) of four wild great tit populations: Westerheide (WH), Wytham Woods (WW), Boshoek (BH), and Lauwersmeer (LM). Box plots indicate medians and the 10th, 25th, 75th and 90th percentiles; 5 th and 95th percentiles are indicated by the filled circles; the open circles indicate the population means.

ID-/- genotypes for this analysis (following Fidler et al. 2007; this equates to a dominant ID- genetic model). We could not confirm the previously reported SNP830 $\times$ ID15 interaction (dominant-T model for SNP830, following Fidler et al. (2007); $\Delta$ deviance $<0.001$, d.f. $=1$, $P=0.99$; see Fig. S1, Supporting Information).

Fig. 2 Proportions of DRD4 SNP830 (A) and ID15 (B) genotypes in four wild great tit populations: Westerheide $(\mathrm{WH})$, Lauwersmeer (LM), Boshoek (BH) and Wytham Woods (WW).

Given that the effect of SNP830 genotype was not different between Fidler et al.'s and our data for this population, we pooled the two datasets to increase the statistical power in further analyses.

A subsequent analysis combining the data from all four populations showed the SNP830 genotype $\times$ population interactions (population d.f. $=3$ ) to be significant for both the additive and dominant-T genetic models (Table 2), but there was no significant overall main effect of SNP830 genotype on exploratory behaviour.

Exploratory behaviour was not significantly associated with ID15 genotype in any of the populations (all $P>0.32$; see Table S4, Supporting Information). Although Fidler and colleagues also found no main effect of ID15, they reported a significant interaction effect of the SNP830 and ID15 genotypes. Therefore, we also tested for such an interaction in our data on the Westerheide for which there was a significant main effect of SNP830. As the homozygote ID15-/- genotype was extremely rare (Fig. 2), we pooled the ID+/- and

\section{Discussion}

We investigated the association between exploratory behaviour and polymorphisms in the DRD4 orthologue in four wild great tit populations. To our knowledge, this is one of the most extensive studies of gene variants influencing personality-related traits in free-living animals to date (see also Trefilov et al. 2000; Krawczak et al. 2005), and the first to compare different wild populations. Fidler et al. (2007) recently identified the orthologue of the human DRD4 gene in the great tit and discovered a significant association between the SNP830 in exon 3 of this gene and variation in exploratory behaviour, both in captive selection lines and handraised birds from a wild population (Westerheide, The Netherlands). Our study confirms this association in an independent sample of free-living individuals from the same population. Additional analyses showed that the strength of the association was not different between Fidler et al.'s hand-raised birds and the free-living birds from this population tested in our study. Separate analyses for three other wild great tit populations (Lauwers- 

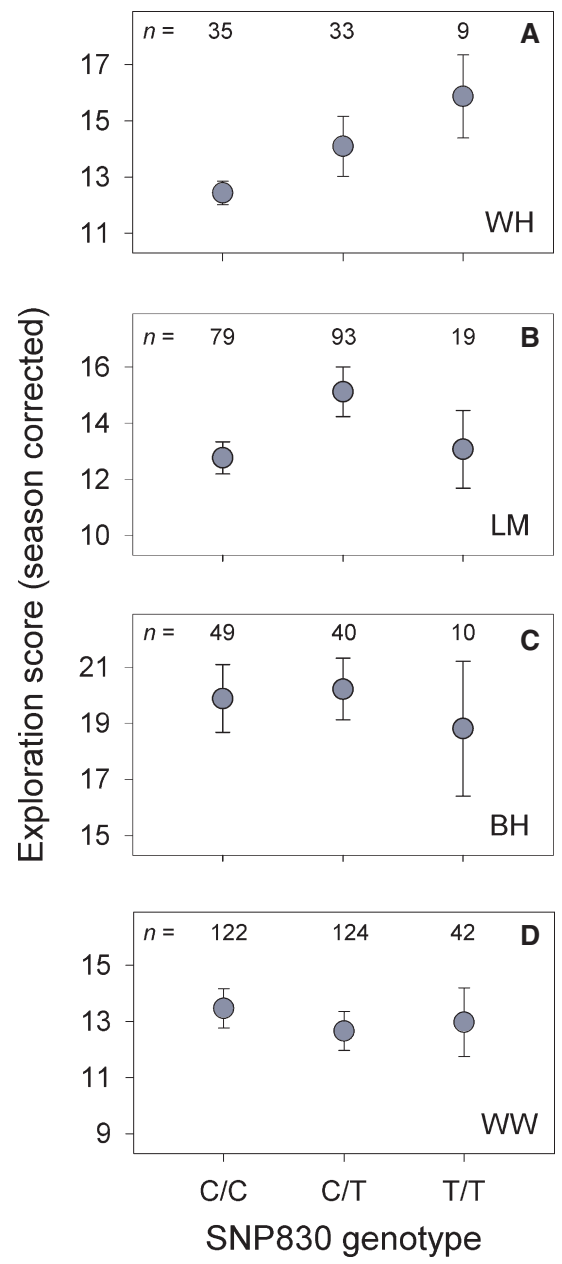

Fig. 4 Exploration scores (corrected for seasonal trend; means with standard errors) of wild great tits of four populations in relation to the DRD4 SNP830 genotype. Exploration scores were significantly associated with SNP830 genotype in Westerheide (See Table 1).

Table 1 Associations between exploratory behaviour and DRD4 SNP830 genotype in four wild great tit populations

\begin{tabular}{llll}
\hline & $\begin{array}{l}\Delta \\
\text { deviance } \\
\left(\chi^{2}{ }_{1}\right)\end{array}$ & $P$ & $\begin{array}{l}\text { Total } \\
\text { variance } \\
\text { explained }\end{array}$ \\
Population & & & \\
\hline (A) Additive genetic model & & & \\
Westerheide $(n=77)$ & 4.316 & 0.038 & $5.8 \%$ \\
Lauwersmeer $(n=191)$ & 1.473 & 0.22 & $0.8 \%$ \\
Boshoek $(n=99)$ & 0.036 & 0.85 & $0.04 \%$ \\
Wytham Woods $(n=288)$ & 0.352 & 0.55 & $0.1 \%$ \\
(B) Dominant-T genetic model & & & \\
Westerheide $(n=77)$ & 3.480 & 0.062 & $4.5 \%$ \\
Lauwersmeer $(n=191)$ & 3.749 & 0.053 & $1.9 \%$ \\
Boshoek $(n=99)$ & 0.001 & 0.97 & $0.001 \%$ \\
Wytham Woods $(n=288)$ & 0.632 & 0.43 & $0.2 \%$ \\
\hline
\end{tabular}

meer, The Netherlands; Boshoek, Belgium; Wytham Woods, UK) indicated that here the association was absent or very weak and not significant. A difference in the strength of the association between SNP830 and exploratory behaviour between the Westerheide and the other populations was also indicated by a significant interaction between the SNP830 genotype and the origin of the data. Our data furthermore show that the association of the SNP830 genotype and exploratory behaviour is independent of the sex of individuals. We could not confirm the influence of the ID15 polymorphism on exploratory behaviour in interaction with the SNP830 as reported by Fidler et al. (2007). This suggests that the previously found effect may have been a false positive result, also because the biological interpretation of this interaction is currently unclear (see Fidler et al. 2007 for discussion).

\section{Effect of SNP830 genotype}

The SNP830 genotype explained $4.5-5.8 \%$ and 5.6-6.0\% of the total variation in exploratory behaviour in our and Fidler et al.'s (2007) studies on the Westerheide population, respectively. From a statistical viewpoint these are small effects, which are difficult to detect and require large sample sizes to prevent strongly increased rates of false negative results (type II errors). From a genetic viewpoint, however, these are substantial effects, in particular for a genetic association of a complex trait like exploratory behaviour or personality (Munafò et al. 2008). Such complex traits are most likely to be influenced by a large number of genes which each have small effects (e.g. Gudbjartsson et al. 2008; Lettre et al. 2008; Weedon et al. 2008). In addition, environmental factors often have a strong influence on these types of trait, as is illustrated by the heritability values for exploratory behaviour in great tits which are between 0.22-0.54 (Dingemanse et al. 2002; Drent et al. 2003; Quinn et al. 2009). In line with these considerations, a recent meta-analysis of DRD4 association studies in humans (Munafò et al. 2008) concluded that a $\mathrm{SNP}$ in the promoter region (C-521T) explained at most $3 \%$ of the variation in novelty seeking and impulsivity.

Although we found no significant associations between SNP830 and exploratory behaviour for the other three populations when separately analysed, we cannot yet exclude the possibility of small effects of SNP830 genotype in some of them. For example, for the Lauwersmeer population, the dominant- $\mathrm{T}$ model was marginally nonsignificant and explained $1.9 \%$ of the total variance in exploratory behaviour, which would be non-negligible as a genetic effect (e.g. Gudbjartsson et al. 2008; Lettre et al. 2008; Weedon et al. 2008). However, such small effects would make it impossible to 
Table 2 Associations between exploratory behaviour and the DRD4 SNP830 genotype of wild great tits and its interaction with study population. This study's and Fidler et al.'s (2007) data for Westerheide were pooled; Lauwersmeer, Boshoek and Wytham Woods populations were included separately (population d.f. $=3 ; n=746$ ). Significance of the population and the SNP830 genotype $\times$ population terms was assessed by adding them sequentially to the model. Note that the main effects of population were nonsignificant, because exploration scores were standardized for each population

\begin{tabular}{|c|c|c|c|c|c|c|}
\hline \multirow[b]{2}{*}{ Genetic model } & \multicolumn{2}{|l|}{ SNP830 } & \multicolumn{2}{|l|}{ Population } & \multicolumn{2}{|c|}{ SNP830 $\times$ Population } \\
\hline & $\Delta$ deviance $\left(\chi_{1}^{2}\right)$ & $P$ & $\Delta$ deviance $\left(\chi_{3}^{2}\right)$ & $P$ & $\Delta$ deviance $\left(\chi_{3}^{2}\right)$ & $P$ \\
\hline Additive & 2.112 & 0.15 & 0.032 & 1.00 & 8.217 & 0.042 \\
\hline Dominant-T & 3.202 & 0.074 & 0.037 & 1.00 & 9.156 & 0.027 \\
\hline
\end{tabular}

use the SNP830 as a genetic marker for personality phenotype, at least for individual variation in exploration behaviour as we measured it, and will make it very challenging to detect current selection on the SNP830 gene variants in the wild. The identification of additional genes influencing personality variation in these populations would enable us to explain a larger proportion of the individual behavioural variation, and may further aid the investigation of the evolutionary processes maintaining this variation. A candidate gene approach may be a suitable method for identifying such additional loci in the great tit (Fitzpatrick et al. 2005; Steinmeyer et al. 2009), which will be greatly facilitated by the publication of the chicken (ICGSC 2004) and zebra finch genomes (http://www.songbirdgenome. org). Alternatively, a large number of SNPs could be used to construct a genetic linkage map for the great tit, and subsequently identify genome regions that associate with variation in exploratory behaviour (van Bers et al. in press).

Between-population differences in the strength of the associations are commonly found in genetic association studies, and may be due to a combination of type I and II errors, as well as genuine population-specific effects (Hirschhorn et al. 2002; Eisenberg et al. 2008). It is not unlikely that in different populations different underlying genetic mechanisms and gene variants contribute to the observed phenotypic variance as a result of, for example, divergent selective pressures or different mutations (Hedrick 2006). The DRD4 SNP830 polymorphism may furthermore be interacting with variants of other genes that may occur at different frequencies in the different populations. Also environmental differences between populations may modify the genetic effects (i.e. gene-environment interaction), as was for example recently documented for the expression of various personality traits in sticklebacks in relation to the presence or absence of predators in their environment (Dingemanse et al. 2009).

At this stage the underlying genetic model of the influence of SNP830 on exploratory behaviour is specu- lative. Our data of the Westerheide population provide both support for an additive genetic model $(P=0.038$; $5.8 \%$ of total variance explained; Table $1 \mathrm{~A})$ and a dominant-T model $(P=0.062 ; 4.5 \%$ of total variance explained; Table 1B). The association pattern in the Lauwersmeer population may even suggest an overdominant model with the $\mathrm{C} / \mathrm{T}$ heterozygotes having the highest mean exploration scores (Fig. 4), although this model was not formally significant ( $\Delta$ deviance $=5.098$, d.f. $=2, P=0.078$ ). It remains uncertain how the effect of the SNP830 comes about, because the polymorphism is synonymous, not leading to a difference in protein structure (Fidler et al. 2007). However, there is growing evidence that synonymous polymorphisms can influence transcription, splicing, mRNA stability and translation, in general (Chamary et al. 2006), and in dopamine receptors, in particular (Duan et al. 2003). Alternatively, the SNP830 may be linked to another functional polymorphism (see Fidler et al. (2007) for a further discussion). For example, Flisikowski et al. (2009) recently showed the presence of linkage disequilibrium between a $D R D 4$ polymorphism and variants of a neighbouring gene involved in the regulation of the serotonergic system ( $D E A F 1$, encoding deformed epidermal auto regulatory factor one) in chickens. In case of linkage disequilibrium of the SNP830 polymorphism with another functional polymorphism, phase differences could potentially exist between populations, which could lead to population differences in the relationship between SNP830 genotype and exploratory behaviour. To further explore this possibility, additional sequencing of the flanking regions of the DRD4 gene for individuals from the different populations is needed.

The frequencies of the SNP830 and ID15 genotypes were very similar between the four populations investigated. One may have expected differences in genotype frequencies in the samples of the different populations due to spatio-temporal variation in the selection on certain personality types (Dingemanse et al. 2004; Both et al. 2005) and associated DRD4 genotypes. Also the differences in magnitude of the exploration scores 
between the populations (Fig. 3) might have suggested population differences in SNP830 genotype frequencies. Nevertheless, genotype frequencies did not differ between the populations (in which birds were also measured in different years, see Table S1, Supporting Information). This might be a result of frequency-dependent selection on the different personality types, which could be driven by the outcome of social or ecological interactions between individuals of different personality type (Dingemanse \& de Goede 2004; Both et al. 2005; van Oers $e t$ al. 2008). In case frequency-dependent selection is driven by the same social and ecological processes in the different populations, this could potentially stabilize the frequencies of $D R D 4$ variants at equal proportions in all populations. Alternatively, frequencies of DRD4 genotypes may not differ between populations due to substantial gene flow between them. Obtaining more information on the genetic distance between the studied populations as well as the presence of genetic structure within them based on neutral genetic markers is important for resolving this issue. There is some evidence to suggest that European mainland great tit populations may be genetically relatively similar, while UK great tits may show limited genetic differentiation from the mainland populations, although there is probably also substantial gene flow from the mainland (Kvist et al. 2007). Genotype frequencies were also not significantly different between the Westerheide sample of Fidler et al. (2007) that was taken in 1998 (Drent et al. 2003) and our sample from 2007 (SNP830: $\chi^{2}=1.195$, d.f. $=2$, $P=0.55$; ID15: $\chi^{2}=4.885$, d.f. $=2, P=0.09$ ). This suggests that genotype frequencies remained similar over multiple generations in the Westerheide population.

\section{Measuring exploratory behaviour}

It is important to mention that our methods for measuring exploratory behaviour were different from the procedures followed by Fidler et al. (2007) in some respects. (i) Fidler and colleagues used a compound measure of exploratory behaviour which included the exploration of a novel test chamber and the approach to novel objects (for more details see Drent et al. 2003), whereas our measure only included exploration of a novel test chamber (following Dingemanse et al. 2002). (ii) The birds studied by Fidler and colleagues were raised under standard laboratory conditions, whereas the birds in our study were caught in the wild one day before being tested. (iii) The birds of Fidler and colleagues were tested at a maximum age of around 35-50 days after hatching (Drent et al. 2003), whereas we included individuals of up to 1 year old. The inclusion of older individuals that were not raised under standard conditions is expected to increase the environmental component of the variation in explor- atory behaviour. Indeed, heritability estimates for personality variation measured in captive great tits bred under standard conditions (selection experiment: realized $h^{2}=0.54$; Drent et al. 2003) appeared somewhat higher than the estimates from great tits in the wild (parent-offspring regressions and sibling analysis: $h^{2}=0.22-0.41$; Dingemanse et al. 2002; 'animal model' methodology using pedigree information: $h^{2}=0.22-0.28$; Quinn et al. 2009). However, despite the methodological differences, our findings for the Westerheide population are remarkably similar to the results reported by Fidler and colleagues, both qualitatively and quantitatively (similar effect sizes; no significant interaction term for the two data sets). The observed personality variation in the Westerheide population, and its association with the SNP830 are apparently rather robust and not very much influenced by environmental factors.

Although we found no difference between populations in the frequencies of the DRD4 genotypes, average exploratory scores differed significantly between populations (Fig. 3). These may be real biological differences, but it is also quite possible that slight methodological differences contribute to this effect (such as the slightly different behavioural test protocol for the Wytham Woods population). Even if slight differences in the measurement protocols contributed to differences in the magnitude of observed exploration scores between study populations, we do not expect this to have a major effect on the associations between $D R D 4$ genotype and exploratory behaviour within populations. The variation in the measured exploration scores can be seen as a proxy for between-individual variation in a suite of correlated functional behaviours (see Introduction), including exploration, aggression, risk taking, routine formation and dispersal (Dingemanse et al. 2003), and the betweenindividual variation in these broad behavioural phenotypes should be relatively robust to small modifications in the exact measurement protocol. As discussed above, local circumstances may also differ between populations and may lead to a different distribution of exploratory scores, as a result of phenotypic plasticity or local selection. Further population comparisons could shine light on this, but the most effective means would involve common environment experiments.

\section{Conclusion}

Our data on free-living great tits of the Westerheide study population confirm the findings of Fidler et al. (2007) who reported an association between the SNP830 in the DRD4 gene on exploratory behaviour in handraised great tits from the same wild population. This confirmation of an association between the SNP830 and exploratory behaviour in a wild great tit population 
warrants further research into the influence of this genetic polymorphism on personality variation in wild great tits and it may open the possibility to detect selection on the different SNP830 gene variants in this population. However, in only one of three other wild great tit populations that we investigated we found weak evidence for an association between exploratory behaviour and SNP830. This finding highlights the importance of studying genetic associations in different populations that may differ in their environmental and genetic background. An important future challenge is to gain a better understanding of population differences in the strength of such genetic associations. Furthermore, the identification of additional loci underlying personality variation in these wild populations is needed to explain a larger proportion of the observed behavioural variation, and may aid in further improving our understanding of the evolutionary processes maintaining such variation.

\section{Acknowledgements}

We thank the people who assisted with catching and testing the birds; for Boshoek: J. Elst, for Lauwersmeer: C. Both, T. Dijkstra, M. Keiser, S. Michler, M. Nicolaus, J. Oldenburger, S. van Schie and R. Ubels, for Westerheide: E. Fucikova and P. de Goede, and for Wytham Woods: S. Bouwhuis, D. Cram, H. Griffith, J. Carpenter, M. Wood, A. Gosler and D. Wilson. We thank Alexander Girg for carrying out most of the ID15 genotyping work. We are very grateful to Matthias Riemenschneider and Patricia Friedrich of the Clinic for Psychiatry and Psychotherapy of the Technical University, Munich, for their help with the SNP830 genotyping and the use of their laboratory facilities. Oscar Langevoord prepared the figure with the locations of the great tit study populations. We thank three anonymous reviewers for their constructive comments. Permission for the transport, short-term housing and personality testing of the birds was granted by the Animal Experiment Committees of the KNAW (Westerheide population) and the University of Groningen (Lauwersmeer), by the Belgian Ringing Scheme and the University of Antwerp (Boshoek), and by Natural England (Wytham Woods). This work was supported by the Max Planck Society (MPG), the Natural Environment Research Council (NERC), a Marie Curie Intra-European Fellowship (MEIF-CT-2006-040639 to ML), and the Alexander von Humboldt Foundation (Fellowship to PK).

\section{References}

Bailey JN, Breidenthal SE, Jorgensen MJ, McCracken JT, Fairbanks LA (2007) The association of DRD4 and novelty seeking is found in a nonhuman primate model. Psychiatric Genetics, 17, 23-27.

van Bers NEM, van Oers K, Kerstens HHD et al. (2010) Genome-wide SNP detection in the great tit Parus major using high throughput sequencing. Molecular Ecology In press.
Biro PA, Stamps JA (2008) Are animal personality traits linked to life-history productivity? Trends in Ecology \& Evolution, 23, 361-368.

Both C, Dingemanse NJ, Drent PJ, Tinbergen JM (2005) Pairs of extreme avian personalities have highest reproductive success. Journal of Animal Ecology, 74, 667-674.

Bouwman KM, Quinn JL, van Overveld $\mathrm{T}$ et al. (submitted) Individual variation in behavioural plasticity across four passerine populations..

Chamary JV, Parmley JL, Hurst LD (2006) Hearing silence: non-neutral evolution at synonymous sites in mammals. Nature Reviews Genetics, 7, 98-108.

Charmantier A, McCleery RH, Cole LR, Perrins C, Kruuk LEB, Sheldon BC (2008) Adaptive phenotypic plasticity in response to climate change in a wild bird population. Science, 320, 800-803.

Dall SRX, Houston AI, McNamara JM (2004) The behavioural ecology of personality: consistent individual differences from an adaptive perspective. Ecology Letters, 7, 734-739.

Dingemanse NJ, de Goede P (2004) The relation between dominance and exploratory behavior is context-dependent in wild great tits. Behavioral Ecology, 15, 1023-1030.

Dingemanse NJ, Réale D (2005) Natural selection and animal personality. Behaviour, 142, 1159-1184.

Dingemanse NJ, Both C, Drent PJ, van Oers K, van Noordwijk AJ (2002) Repeatability and heritability of exploratory behaviour in great tits from the wild. Animal Behaviour, 64, 929-938.

Dingemanse NJ, Both C, van Noordwijk AJ, Rutten AL, Drent PJ (2003) Natal dispersal and personalities in great tits (Parus major). Proceedings of the Royal Society of London. Series B: Biological Sciences, 270, 741-747.

Dingemanse NJ, Both C, Drent PJ, Tinbergen JM (2004) Fitness consequences of avian personalities in a fluctuating environment. Proceedings of the Royal Society of London. Series B: Biological Sciences, 271, 847-852.

Dingemanse NJ, Van der Plas F, Wright J et al. (2009) Individual experience and evolutionary history of predation affect expression of heritable variation in fish personality and morphology. Journal of Animal Ecology, 76, 1128-1138.

Drent PJ, van Oers K, van Noordwijk AJ (2003) Realized heritability of personalities in the great tit (Parus major). Proceedings of the Royal Society of London. Series B: Biological Sciences, 270, 45-51.

Duan JB, Wainwright MS, Comeron JM et al. (2003) Synonymous mutations in the human dopamine receptor D2 (DRD2) affect mRNA stability and synthesis of the receptor. Human Molecular Genetics, 12, 205-216.

Duckworth RA, Badyaev AV (2007) Coupling of dispersal and aggression facilitates the rapid range expansion of a passerine bird. Proceedings of the National Academy of Sciences of the United States of America, 104, 15017-15022.

Eisenberg DTA, Campbell B, Gray PB, Sorenson MD (2008) Dopamine receptor genetic polymorphisms and body composition in undernourished pastoralists: an exploration of nutrition indices among nomadic and recently settled Ariaal men of northern Kenya. BMC Evolutionary Biology, 8, 173.

Ellegren H, Sheldon BC (2008) Genetic basis of fitness differences in natural populations. Nature, 452, 169-175. 
Fidler AE, van Oers K, Drent PJ, Kuhn S, Mueller JC, Kempenaers B (2007) DRD4 gene polymorphisms are associated with personality variation in a passerine bird. Proceedings of the Royal Society of London. Series B: Biological Sciences, 274, 1685-1691.

Fitzpatrick MJ, Ben-Shahar Y, Smid HM, Vet LEM, Robinson GE, Sokolowski MB (2005) Candidate genes for behavioural ecology. Trends in Ecology \& Evolution, 20, 96-104.

Flisikowski K, Schwarzenbacher H, Wysocki M et al. (2009) Variation in neighbouring genes of the dopaminergic and serotonergic systems affects feather pecking behaviour of laying hens. Animal Genetics, 40, 192-199.

Gosling SD (2001) From mice to men: what can we learn about personality from animal research? Psychological Bulletin, 127, $45-86$.

Greenberg R (1990) Feeding neophobia and ecological plasticity-a test of the hypothesis with captive sparrows. Animal Behaviour, 39, 375-379.

Griffiths R, Double MC, Orr K, Dawson RJG (1998) A DNA test to sex most birds. Molecular Ecology, 7, 1071-1075.

Groothuis TGG, Carere C (2005) Avian personalities: characterization and epigenesis. Neuroscience and Biobehavioral Reviews, 29, 137-150.

Gudbjartsson DF, Walters GB, Thorleifsson G et al. (2008) Many sequence variants affecting diversity of adult human height. Nature Genetics, 40, 609-615.

Harfmann Short K, Petren K (2008) Boldness underlies foraging success of invasive Lepidodactylus lugubris geckos in the human landscape. Animal Behaviour, 76, 429-437.

Hedrick PW (2006) Genetic polymorphism in heterogeneous environments: the age of genomics. Annual Review of Ecology Evolution and Systematics, 37, 67-93.

Hejjas K, Vas J, Topal J et al. (2007) Association of polymorphisms in the dopamine $\mathrm{D} 4$ receptor gene and the activity-impulsivity endophenotype in dogs. Animal Genetics, 38, 629-633.

Hirschhorn JN, Lohmueller K, Byrne E, Hirschhorn K (2002) A comprehensive review of genetic association studies. Genetics in Medicine, 4, 45-61.

Hollander FA, Van Overveld T, Tokka I, Matthysen E (2008) Personality and nest defence in the Great Tit (Parus major). Ethology, 114, 405-412.

[ICGSC] International Chicken Genome Sequencing Consortium (2004) Sequence and comparative analysis of the chicken genome provide unique perspectives on vertebrate evolution. Nature, 432, 695-716.

James AS, Groman SM, Seu E, Jorgensen M, Fairbanks LA, Jentsch JD (2007) Dimensions of impulsivity are associated with poor spatial working memory performance in monkeys. Journal of Neuroscience, 27, 14358-14364.

Jenni L, Winkler R (1994) Moult and Ageing in European Passerines. Academic Press, London.

Krawczak M, Trefilov A, Berard J et al. (2005) Male reproductive timing in rhesus macaques is influenced by the 5HTTLPR promoter polymorphism of the serotonin transporter gene. Biology of Reproduction, 72, 1109-1113.

Kvist L, Arbabi T, Päckert M, Orell M, Martens J (2007) Population differentiation in the marginal populations of the great tit (Paridae: Parus major). Biological Journal of the Linnean Society, 90, 201-210.
Lettre G, Jackson AU, Gieger C et al. (2008) Identification of ten loci associated with height highlights new biological pathways in human growth. Nature Genetics, 40, 584591.

Martin LB, Fitzgerald L (2005) A taste for novelty in invading house sparrows, Passer domesticus. Behavioral Ecology, 16, 702-707.

McCleery RH, Pettifor RA, Armbruster P, Meyer K, Sheldon BC, Perrins CM (2004) Components of variance underlying fitness in a natural population of the great tit Parus major. American Naturalist, 164, E62-E72.

McGue M, Bouchard TJ (1998) Genetic and environmental influences on human behavioral differences. Annual Review of Neuroscience, 21, 1-24.

McNamara JM, Stephens PA, Dall SRX, Houston AI (2009) Evolution of trust and trustworthiness: social awareness favours personality differences. Proceedings of the Royal Society of London B, 276, 605-613.

Mitchell-Olds T, Willis JH, Goldstein DB (2007) Which evolutionary processes influence natural genetic variation for phenotypic traits? Nature Reviews Genetics, 8, 845-856.

Momozawa Y, Takeuchi Y, Kusunose R, Kikusui T, Mori Y (2005) Association between equine temperament and polymorphisms in dopamine D4 receptor gene. Mammalian Genome, 16, 538-544.

Munafò MR, Clark TG, Moore LR, Payne E, Walton R, Flint J (2003) Genetic polymorphisms and personality in healthy adults: a systematic review and meta-analysis. Molecular Psychiatry, 8, 471-484.

Munafò MR, Yalcin B, Willis-Owen SA, Flint J (2008) Association of the dopamine D4 receptor (DRD4) gene and approach-related personality traits: meta-analysis and new data. Biological Psychiatry, 63, 197-206.

Netter P (2006) Dopamine challenge tests as an indicator of psychological traits. Human Psychopharmacology-Clinical and Experimental, 21, 91-99.

Nicolaus M, Michler SPM, Ubels R et al. (2009) Sex-specific effects of altered competition on nestling growth and survival: an experimental manipulation of brood size and sex ratio. Journal of Animal Ecology, 78, 414-426.

van Oers K, de Jong G, Drent PJ, van Noordwijk AJ (2004a) A genetic analyses of avian personality traits; correlated response to artificial selection. Behavior Genetics, 34, 611619.

van Oers K, Drent PJ, de Goede P, van Noordwijk AJ (2004b) Realized heritability and repeatability of risk-taking behaviour in relation to avian personalities. Proceedings of the Royal Society B: Biological Sciences, 271, 65-73.

van Oers K, de Jong G, van Noordwijk AJ, Kempenaers B, Drent PJ (2005) Contribution of genetics to the study of animal personalities: a review of case studies. Behaviour, 142, 1185-1206.

van Oers K, Drent PJ, Dingemanse NJ, Kempenaers B (2008) Personality is associated with extrapair paternity in great tits, Parus major. Animal Behaviour, 76, 555-563.

Postma E, van Noordwijk AJ (2005) Gene flow maintains a large genetic difference in clutch size at a small spatial scale. Nature, 433, 65-68.

Quinn JL, Patrick S, Bouwhuis S, Wilkin TA, Sheldon BC (2009) Heterogeneous selection on a heritable temperament 
trait in a complex environment. Journal of Animal Ecology, 78, 1203-1215.

Rasbash J, Steele F, Browne W, Prosser B (2004) A User's Guide to MLwiN. Institute of Education, London.

Réale D, Reader SM, Sol D, McDougall PT, Dingemanse NJ (2007) Integrating animal temperament within ecology and evolution. Biological Reviews, 82, 291-318.

Savitz JB, Ramesar RS (2004) Genetic variants implicated in personality: a review of the more promising candidates. American Journal of Medical Genetics B, 131, 20-32.

Shultz S, Bradbury RB, Evans KL, Gregory RD, Blackburn TM (2005) Brain size and resource specialization predict longterm population trends in British birds. Proceedings of the Royal Society B: Biological Sciences, 272, 2305-2311.

Sih A, Bell A, Johnson JC (2004a) Behavioral syndromes: an ecological and evolutionary overview. Trends in Ecology $\mathcal{E}$ Evolution, 19, 372-378.

Sih A, Bell AM, Johnson JC, Ziembam RE (2004b) Behavioral syndromes: an integrative overview. Quarterly Review of Biology, 79, 241-277.

Sol D, Timmermans S, Lefebvre L (2002) Behavioural flexibility and invasion success in birds. Animal Behaviour, 63, 495-502.

Steinmeyer C, Mueller JC, Kempenaers B (2009) Search for informative polymorphisms in candidate genes: clock genes and circadian behaviour in blue tits. Genetica, 136, 109-117.

Trefilov A, Berard J, Krawczak M, Schmidtke J (2000) Natal dispersal in rhesus macaques is related to serotonin transporter gene promoter variation. Behavior Genetics, 30, 295-301.

Verbeek MEM, Drent PJ, Wiepkema PR (1994) Consistent individual differences in early exploratory behaviour of male great tits. Animal Behaviour, 48, 1113-1121.

Verbeek MEM, Boon A, Drent PJ (1996) Exploration, aggressive behavior and dominance in pair-wise confrontations of juvenile male great tits. Behaviour, 133, 945-963.

Walsh PS, Metzger DA, Higuchi R (1991) Chelex-100 as a medium for simple extraction of DNA for PCR-Based typing from forensic material. BioTechniques, 10, 506-513.

Weedon MN, Lango H, Lindgren CM et al. (2008) Genomewide association analysis identifies 20 loci that influence adult height. Nature Genetics, 40, 575-583.

Wilson DS (1998) Adaptive individual differences within single populations. Philosophical Transactions of the Royal Society of London. Series B: Biological Sciences, 353, 199-205.

Wolf M, van Doorn GS, Leimar O, Weissing FJ (2007) Lifehistory trade-offs favour the evolution of animal personalities. Nature, 447, 581-584.

Wolf M, van Doorn GS, Weissing FJ (2008) Evolutionary emergence of responsive and unresponsive personalities. Proceedings of the National Academy of Sciences of the United States of America, 105, 15825-15830.
This paper is the product of a very successful and large-scale international collaborative effort of the researchers involved. Peter Korsten is an evolutionary ecologist studying selection and genetic variation in wild populations. Jakob Mueller is interested in evolutionary genomics of behavioural traits and contributed to study design, data analysis and manuscript writing. Christine Hermannstädter is a technical assistant who contributed via her laboratory skills. Miriam Liedvogel is a molecular ecologist who independently genotyped the DRD4 for the Wytham population. Bart Kempanaers is head of the Department Behavioural Ecology and Evolutionary Genetics and initiated this work. The other coauthors are evolutionary ecologists who contributed personality data and samples from their study population of great tits, and who commented on the manuscript.

\section{Supporting information}

Additional supporting information may be found in the online version of this article.

Table S1 Wild great tit populations investigated for association of exploratory behaviour and DRD4 SNP830 and ID15 genotype ( $n=666$ individuals)

Table S2 None of the four great tit populations deviated significantly from Hardy-Weinberg equilibrium for the DRD4 SNP830 and ID15 polymorphisms

Table S3 No main effects of the sex of individuals or genotype $\times$ sex interaction effects on exploration scores in four populations of wild great tits. Significance of the sex and the SNP830 genotype $\times$ sex terms was assessed by adding them sequentially to the model

Table S4 Associations between exploratory behaviour and DRD4 ID15 genotype in four wild great tit populations

Fig. S1 Exploration scores (corrected for seasonal trend; means with standard errors) of wild great tits of the Westerheide population in relation to the DRD4 SNP830 and ID15 genotypes. Groups represent individuals either with or without the SNP830 T allele and either with or without the ID15- allele (following Fidler et al. 2007). The interaction between the SNP830 and the ID15 polymorphisms was not significant ( $\Delta$ deviance $<0.001$, d.f. $=1, P=0.99$ ).

Please note: Wiley-Blackwell are not responsible for the content or functionality of any supporting information supplied by the authors. Any queries (other than missing material) should be directed to the corresponding author for the article. 